\title{
Comparison of radiometric and gas capture system for blood cultures
}

\author{
ANNA KING, G BONE, I PHILLIPS \\ From the Department of Microbiology, St Thomas's Hospital, London
}

SUMMARY A single bottle blood culture system that detects the gas produced by micro-organisms from substrates in a specially formulated medium was compared with the Bactec radiometric system. A total of 725 blood samples collected from patients with suspected bacteraemia yielded 75 clinically important isolates. Fifty five $(73 \%)$ of these cultures were detected by both methods, 16 $(21 \%)$ by only the Bactec system, and four $(5 \%)$ by only the Oxoid system. No significant differences were found between the two systems after allowance was made for the different volume of sample inoculated into each system.

For many years microbiologists have aimed at developing an efficient blood culture system for the rapid isolation of organisms from patients with suspected bacteraemia. The composition and number of different types of media used for such isolation have been controversial subjects. In the 1972 British blood culture survey nutrient broth and glucose broth were the most popular media for aerobic culture and many types of media were used for anaerobic culture, including Robertson's cooked meat, thioglycolate broth (Difco), and Brewer's thioglycolate broth (Southern Group). ${ }^{1}$

During the early 1970s rapid methods for the diagnosis of bacteraemia were investigated, including the use of electrical impedance, gas-liquid chromatography, and radiometric techniques. The radiometric investigations led to the introduction of the method used in the Bactec $460,{ }^{2}$ which has been the routine system used at St Thomas's Hospital for the past three years. Oxoid have recently developed a single bottle blood culture system that measures production of gas from a specially formulated medium capable of supporting the growth of both aerobic and anaerobic organisms. In this study we compared the Bactec system with the prototype Oxoid system.

\section{Material and methods}

The prototype Oxoid system consists of a phial containing $36.5 \mathrm{ml}$ of culture broth that is sealed with a thick rubber septum to maintain the initial vacuum and give support to the reservoir unit, which is inserted into the phial after inoculation with the blood sample. The reservoir unit consists of a hypodermic needle connected to a sterile plastic chamber with a calibrating ring mark and a screw cap with a central vent plugged with cotton wool. Gas produced by growing organisms increases the pressure in the phials, and medium is forced up the needle into the reservoir.

Blood samples were collected by the laboratory staff from patients at St Thomas's Hospital. A total of $15 \mathrm{ml}$ of blood was collected for each culture and divided equally into three bottles: one containing Bactec 6B medium (enriched tryptic soy broth) for aerobic culture; another containing Bactec 7D medium (pre-reduced tryptic soy broth) for anaerobic culture; and an Oxoid bottle.

Bactec aerobic bottles were placed on a mechanical shaker at $37^{\circ} \mathrm{C}$ for 24 hours and left static for the rest of the incubation period. The bottles were tested once during the afternoon of day 1 (day of collection) if collected before midday, twice on day 2 , and then daily up to. day 7 . Bactec anaerobic bottles were static throughout the incubation period. They were tested once each day from day 2 to day 7 .

All Bactec bottles were incubated in Bactec preloading racks and tested on a model 460 instrument for the production of ${ }^{14}$ carbon dioxide $\left({ }^{14} \mathrm{CO}_{2}\right)$. They were considered to be positive if the growth index reading exceeded that of the preset threshold values of 35 for aerobic bottles and 25 for anaerobic bottles. All bottles were inspected for obvious evidence of growth before they were placed on the machine. To decrease the possibility of cross contamination cultures that were obviously positive were not sampled for ${ }^{14} \mathrm{CO}_{2}$.

For the first two weeks of the trial all Oxoid bottles 
were static throughout the incubation period. The method was then changed as some bottles with obvious growth had failed to give positive reactions; they were mixed gently twice daily throughout the incubation period for the rest of the study. The bottles were examined for increased pressure in the afternoon of day 1 and twice daily for up to seven days.

A Gram stained smear was examined from all bottles with positive reactions. Subcultures of the samples were made on to two blood agar plates, one of which was incubated in air with $10 \%$ carbon dioxide and the other in a mixture of nitrogen $(80 \%)$, carbon dioxide $(10 \%)$, and hydrogen $(10 \%)$ in an anaerobic chamber (Don Whitley). Further subcultures and sensitivity and biochemical tests were performed as appropriate from the result of the Gram stained smear.

Experimental inoculation of bottles was performed to try to establish the most satisfactory method of incubation of the Oxoid bottles and test the ability of the Oxoid medium to support the growth of organisms that were not isolated from clinical samples during the trial period. Organisms that were isolated only from one system during the trial were also reinoculated into both systems. Five bottles were inoculated with each organism: one Bactec $6 \mathrm{~B}$, one Bactec 7D, and three Oxoid bottles. Blood $(5 \mathrm{ml})$ from healthy volunteers was added to each bottle, which was then inoculated with a small number of organisms (four-50 colony forming units). The Bactec bottles were incubated and tested as before. All Oxoid bottles were incubated at $37^{\circ} \mathrm{C}$, one was left static throughout the incubation period, one was mixed gently twice daily, and the third was shaken for the first 24 hours and left static for the rest of the incubation period. All Oxoid bottles were examined twice daily for seven days. All bottles that were negative at the end of the seven day incubation period were subcultured on to appropriate media.

\section{Results}

A total of 725 blood cultures collected from 679 patients with suspected bacteraemia yielded 75 $(10.3 \%)$ clinically significant isolates. The Bactec aerobic bottles yielded positive cultures in $63(84 \%)$ cases and the Bactec anaerobic bottle in $52(69 \%)$ cases, accounting for a total of $71(94 \%)$ of the positive cultures. The Oxoid bottles yielded positive cultures in $61(81 \%)$ cases. A single organism was isolated from 69 positive cultures (Table 1), and more than one organism was isolated from six cultures (Table 2).

False positive results (a reading above the threshold value in Bactec bottles and evidence of increased pressure in the Oxoid bottle but no growth of organisms) were recorded in $13(1.8 \%)$ Bactec aerobic bottles, one $(0.13 \%)$ Bactec anaerobic bottle, and one $(0 \cdot 13 \%)$ Oxoid bottle. In most of the Bactec aerobic bottles giving false positive readings there was evidence on Gram staining of increased numbers of white blood cells.

The rate of contamination was similar in both systems; Bactec aerobic bottles were contaminated on $11(1.5 \%)$ occasions, Bactec anaerobic bottles on four $(0 \cdot 4 \%)$ and Oxoid bottles on eight $(1 \cdot 1 \%)$. Staphy-

Table 1 Positive blood cultures yielding single organisms

\begin{tabular}{|c|c|c|c|c|}
\hline & \multirow[t]{2}{*}{ Total } & \multicolumn{2}{|c|}{ Bactec bottles } & \multirow[t]{2}{*}{ Oxoid bottles } \\
\hline & & Aerobic & Anaerobic & \\
\hline $\begin{array}{l}\text { Staphylococcus aureus } \\
\text { Staphylococcus epidermidis } \\
\text { Streptococcus pneumoniae } \\
\text { Streptococcus faecalis } \\
\text { Streptococcus salivarius } \\
\text { Streptococcus sanguis } \\
\text { Streptococcus bovis } \\
\text { Streptococcus milleri } \\
\text { Streptococcus mitior } \\
\text { Corynebacterium sp } \\
\text { Escherichia coli } \\
\text { Klebsiella sp } \\
\text { Citrobacter freundii } \\
\text { Citrobacter koseri } \\
\text { Enterobacter cloacae } \\
\text { Proteus mirabilis } \\
\text { Pseudomonar aeruginosa } \\
\text { Haemophilus influenzae } \\
\text { Peptococcus sp } \\
\text { Bacteroides fragilis } \\
\text { Total }\end{array}$ & $\begin{array}{r}11 \\
7 \\
5 \\
5 \\
3 \\
3 \\
2 \\
1 \\
1 \\
2 \\
12 \\
4 \\
3 \\
2 \\
2 \\
1 \\
1 \\
2 \\
1 \\
1 \\
69\end{array}$ & $\begin{array}{r}11 \\
7 \\
4 \\
5 \\
3 \\
3 \\
2 \\
1 \\
1 \\
10 \\
3 \\
3 \\
2 \\
1 \\
2 \\
58\end{array}$ & $\begin{array}{r}10 \\
4 \\
4 \\
4 \\
\\
3 \\
2 \\
\\
\\
1 \\
7 \\
3 \\
2 \\
2 \\
2 \\
1 \\
\\
1 \\
1 \\
1 \\
48\end{array}$ & $\begin{array}{c}11 \\
5 * \\
4 \\
4 \\
3 \\
2 \\
1 \\
1 \\
2 \\
11 \\
4 \\
2 \\
1 \\
2 \\
1 \\
\\
1 * \\
1 \\
1 \\
57\end{array}$ \\
\hline
\end{tabular}

"One isolate showed no indication of increased pressure but grew on terminal subculture. 
Table 2 Positive blood cultures yielding two organisms

\begin{tabular}{|c|c|c|c|}
\hline & \multicolumn{2}{|l|}{ Bactec bottles } & \multirow[t]{2}{*}{ Oxoid bottles } \\
\hline & Aerobic & Anaerobic & \\
\hline $\begin{array}{l}\text { Escherichia coli and Listeria monocytogenes } \\
\text { Branhamella catarrhalis and Fusobacterium nucleatum } \\
\text { Streptococcus faecalis and Escherichia coli } \\
\text { Klebsiella sp and Pseudomonas aeruginosa } \\
\text { Peptococcus sp and Veillonella sp } \\
\text { Proteus mirabilis and Streptococcus faecalis }\end{array}$ & $\begin{array}{l}\text { Both } \\
\text { B catarrhalis } \\
\text { Both } \\
\text { Both } \\
\text { Both }\end{array}$ & $\begin{array}{l}\text { E coli } \\
\text { F nucleatum } \\
\text { Both } \\
\text { Both }\end{array}$ & $\begin{array}{l}\text { Both } \\
\text { Both } \\
\text { Both } \\
\text { Both }\end{array}$ \\
\hline
\end{tabular}

Table 3 Day of isolation of organisms in positive culture

\begin{tabular}{lcc}
\hline Day of isolation & \multicolumn{2}{c}{ No of different organisms } \\
\cline { 2 - 3 } & \multicolumn{1}{c}{ Bactec bottles } & Oxoid bottles \\
\hline When Oxoid bottles incubated without mixing \\
2 & 9 & 5 \\
3 & 4 & 5 \\
4 & 2 & 3 \\
5 & & 1 \\
6 & & 1 \\
7 & 25 & 19 \\
When Oxoid bottles were mixed twice daily & 18 \\
2 & 12 & 3 \\
3 & 3 & \\
4
\end{tabular}

Table 4 Isolates from only one of the two systems

\begin{tabular}{|c|c|c|}
\hline & \multicolumn{2}{|c|}{ Isolation from Bactec } \\
\hline & Aerobic & Anaerobic \\
\hline $\begin{array}{l}\text { Isolates from Bactec } \\
\text { but not from Oxoid system: } \\
\text { Escherichia coli } \\
\text { Citrobacter koseri } \\
\text { Citrobacter freundii } \\
\text { Staphylococcus epidermidis } \times 2 \\
\text { Streptococcus faecalis } \\
\text { Streptococcus pneumoniae } \\
\text { Streptococcus salivarius } \times 3 \\
\text { Haemophilus influenzae } \\
\text { Pseudomonas aeruginosa } \times 2 \\
\text { Branhamella catarrhalis } \\
\text { Fusobacterium nucleatum } \\
\text { Klebsiella sp }\end{array}$ & $\begin{array}{l}- \\
\overline{+} \\
+ \\
+ \\
+ \\
+ \\
+ \\
+ \\
+ \\
+ \\
+ \\
+\end{array}$ & $\begin{array}{l}+ \\
+ \\
- \\
- \\
- \\
+ \\
- \\
- \\
- \\
- \\
+ \\
-\end{array}$ \\
\hline $\begin{array}{l}\text { Isolates from Oxoid but not from } \\
\text { Escherichia coli } \\
\text { Streptococcus pneumoniae } \\
\text { Streptococcus milleri } \\
\text { Corynebacterium sp }\end{array}$ & ctec syste & \\
\hline
\end{tabular}

lococcus epidermidis was the contaminating organism in 19 cultures, Bacillus in three, and Propionibacterium in one.

There was a noticeable improvement in the rapidity with which the Oxoid bottles produced a positive reaction when gentle mixing was introduced (Table 3 ); this was noticed particularly in cultures growing staphylococci.

Sixteen isolates, representing 12 species, grew only in the Bactec system, 15 of which were isolated from one bottle, the other being a Streptococcus pneumoniae that grew in both bottles, and four isolates grew only in the Oxoid system (Table 4). When reinoculated eight of these organisms grew in both systems, though not always in all bottles (Table 5). The Corynebacterium sp did not grow in the Bactec aerobic bottle and failed to give a positive reaction in both the static and shaken Oxoid bottles, though it was obviously growing in both and was recovered on subculture. The Fusobacterium nucleatum grew only in the static Oxoid bottle and not in the mixed or shaken bottles, and, as expected, it also failed to grow in the aerobic Bactec bottle. The isolate of Branhamella catarrhalis did not grow in the anaerobic Bactec bottle.

The results were similar for the eight stock cultures inoculated into both systems (Table 6). Neither of the Neisseria spp grew in the Bactec anaerobic bottles, and the Bacteroides fragilis and Clostridium perfringens did not grow in the Bactec aerobic bottles. There was growth in all Oxoid bottles, but again some bottles failed to show evidence of increased pressure. This varied in relation to the conditions of incubation and the species: the isolate of Gardnerella vaginalis did not give a positive reaction in the static bottle; neither of the Neisseria spp produced gas in the mixed bottle and the $N$ gonorrhoeae was also negative in the shaken bottle; and the Clostridium perfringens showed no reaction in either the static or shaken bottle.

\section{Discussion}

These preliminary results of the prototype Oxoid system were encouraging. Neither the Bactec nor Oxoid system was able to detect all the clinically significant isolates. The greater rate of detection in the Bactec system may, in part, be due to twice the volume of blood being inoculated in the Bactec system than in the Oxoid system, especially for patients in whom the number of organisms/ml of blood was low. The finding that most isolates, which did not grow in all bottles, were detected only in one bottle would support this theory. We were perplexed, however, by the three isolates of Streptococcus salivarius (isolated from three separate blood cultures of one patient's 
Table 5 Inoculation of organisms that did not grow in both systems

\begin{tabular}{|c|c|c|c|c|c|c|}
\hline & \multirow{3}{*}{$\begin{array}{l}\text { Inoculum size } \\
\text { (cfu) }\end{array}$} & \multicolumn{5}{|c|}{ Day of isolation in: } \\
\hline & & \multicolumn{2}{|c|}{ Bactec bottle } & \multicolumn{3}{|c|}{ Oxoid bottle } \\
\hline & & Aerobic & Anaerobic & Static & Mixed & Shaken \\
\hline $\begin{array}{l}\text { Staphylococcus epidermidis } \\
\text { Streptococcus salivarius } \\
\text { Corynebacterium sp } \\
\text { Klebsiella sp } \\
\text { Pseudomonas aeruginosa } \\
\text { Haemophilus influenzae } \\
\text { Branhamella catarrhalis } \\
\text { Fusobacterium nucleatum }\end{array}$ & $\begin{array}{r}6 \\
44 \\
6 \\
52 \\
40 \\
24 \\
40 \\
4\end{array}$ & $\begin{array}{l}2 \\
3 \\
2 \\
2 \\
2 \\
2\end{array}$ & $\begin{array}{l}2 \\
2 \\
5 \\
2 \\
3 \\
3 \\
5\end{array}$ & $\begin{array}{l}3 \\
3 \\
4 * \\
2 \\
2 \\
4 \\
5 \\
5\end{array}$ & $\begin{array}{l}3 \\
2 \\
3 \\
2 \\
2 \\
3 \\
5\end{array}$ & $\begin{array}{l}2 \\
2 \\
4^{*} \\
2 \\
3 \\
4 \\
4\end{array}$ \\
\hline
\end{tabular}

CFU $=$ Colony forming units.

* No increase in pressure but obvious growth and organism isolated on subculture.

Table 6 Inoculation of stock cultures of organism not isolated from clinical specimens

\begin{tabular}{|c|c|c|c|c|c|c|}
\hline & \multirow{3}{*}{$\begin{array}{l}\text { Inoculum size } \\
\text { (cfu) }\end{array}$} & \multicolumn{5}{|c|}{ Day of isolation in: } \\
\hline & & \multicolumn{2}{|c|}{ Bactec bottle } & \multicolumn{3}{|c|}{ Oxoid bottle } \\
\hline & & Aerobic & Anaerobic & Static & Mixed & Shaken \\
\hline $\begin{array}{l}\text { Streptococcus pyogenes } \\
\text { Salmonella typhimurium } \\
\text { Candida albicans } \\
\text { Gardnerella vaginalis } \\
\text { Neisseria gonorrhoeae } \\
\text { Neisseria meningitidis } \\
\text { Bacteroides fragilis } \\
\text { Clostridium perfringens }\end{array}$ & $\begin{array}{l}16 \\
48 \\
34 \\
52 \\
24 \\
20 \\
30 \\
12\end{array}$ & $\begin{array}{l}2 \\
2 \\
3 \\
3 \\
6 \\
2\end{array}$ & $\begin{array}{l}2 \\
2 \\
7 \\
3\end{array}$ & $\begin{array}{l}2 \\
2 \\
4 \\
3^{*} \\
5 \\
4 \\
3 \\
4^{*}\end{array}$ & $\begin{array}{l}2 \\
2 \\
4 \\
3 \\
4^{*} \\
4^{*} \\
3 \\
3\end{array}$ & $\begin{array}{l}2 \\
2 \\
3 \\
3 \\
4^{*} \\
5 \\
5 \\
4^{*}\end{array}$ \\
\hline
\end{tabular}

CFU $=$ Colony forming units.

*No increase in pressure but obvious growth and organism isolated on subculture.

blood) and two isolates of Pseudomonas aeruginosa that were detected only in the Bactec aerobic bottles. Although these organisms grew in the Oxoid system when reinoculated in the laboratory, we have no proof that the system can detect these species on primary isolation.

The mixing of the Oxoid bottles certainly improved the detection time during the trial, and this was confirmed in the experiments to establish the best method of incubation. Interestingly, shaking the bottles for the first 24 hours improved the detection time in two cultures only and in six prolonged the detection time so that it was equal to or longer than that in the static bottles. The number of bottles showing no increase in pressure, although the organisms had grown, was also slightly lower in the mixed bottles than in the static and shaken bottles. The isolate of Fusobacterium nucleatum, however, the most fastidious of the anaerobic bacteria we tested for, grew only in the static bottle. This suggests that if mixing were to become the standard method for this system certain anaerobic bacteria may not be detected.

The incidence of false positive results was greatly reduced from that in the Bactec aerobic cultures from patients with severe leucocytosis, and this saved a considerable amount of technical time. Interestingly, a subsequent blood culture from the patient giving the single false positive result in the Oxoid bottle grew Staphylococcus aureus.

In practical terms the Oxoid system was simple to use and recognition of positive bottles was easy. Bottles needed to be inspected soon after removal from the incubator as the level of fluid in the reservoir fell as the temperature decreased and some bottles containing isolates that had produced only small amounts of gas appeared negative. With experience it was possible to predict the organism present, as certain species had distinctive "diagnostic" features. Gram negative organisms tended to produce more gas, and hence the volume of fluid in the reservoir was greater, and, in addition, Pseudomonas aeruginosa produced a froth or layer of scum on the top of the fluid in the reservoir. In contrast, Gram positive organisms produced less gas and hence less fluid in the reservoir, and there was evidence of either lysis or sedimented red cells in the medium in the reservoir.

The screw cap on the reservoir facilitated easy access for samples to be taken for Gram stains and subcultures, and although it was important to inspect all bottles before they were discarded for evidence of 
growth that had not produced a positive reaction, we found routine terminal subculture unnecessary. Advantages of the Oxoid system were that no expensive automated equipment was required and there was no radiation hazard or problems of disposal of radioactive waste. We did, however, find the sterilisation of the discarded bottles tiresome as the plastic reservoir melts when autoclaved, and hence the bottles had to be kept upright and for safety reasons after sterilisation the hypodermic syringe was separated from the bottles before disposal.

A distinct advantage of the Oxoid system was that it could be used to culture blood for both aerobic and anaerobic organisms thus removing the decision of which medium to inoculate on the basis of clinical information when insufficient blood had been collected for inoculation of two bottles.

We found the thick rubber septum, necessary for maintaining a vacuum, difficult to penetrate and occasionally the cotton wool plug imploded. Since the trial with this prototype system, however, there have been design changes that should eradicate some of the problems we experienced. These changes include the replacement of the cotton wool plug with a $0.2 \mu \mathrm{m}$ polytetrafluoroethylene membrane filter, the addition of a rigid plastic sleeve to hold the reservoir unit more securely to the bottle, and, perhaps most importantly, an increase in volume of medium so that the system will now take a total of $10 \mathrm{ml}$ of blood.

In conclusion, we think this system has great potential and recommend a larger study to elucidate the few problems.

\section{References}

${ }^{1}$ Shanson DC. Blood culture techniques. In: Williams JD, ed. Modern topics in infection London: William Heinemann Medical Books, 1978:14-30.

${ }^{2}$ DeBlanc HJ, DeLand F, Wagner HN. Automated radiometric detection of bacteria in 2,967 blood cultures. Appl Microbiol 1971;22:846-9.

Requests for reprints to: Dr Anna King, Department of Microbiology, St Thomas's Hospital Medical School, London SEl 7EH, England. 